\title{
Tendencias en la formación inicial docente
}

\author{
Denise Vaillant, * \\ Jesús Manso**
}

\section{Resumen}

En los últimos años, se han multiplicado los estudios e informes que muestran insatisfacción sobre la calidad de los programas de formación docente. Se hace necesario, hoy más que nunca, preguntarse cómo lograr la mejora de la formación inicial de maestros y profesores, para que ésta tenga real impacto en las aulas. Por esta razón, los autores proponen un análisis y reflexión acerca de una serie de iniciativas identificadas en la literatura. Las reformas e innovaciones presentadas han sido agrupadas en cinco categorías de análisis referidas al establecimiento de estándares en las instituciones formadoras; al uso de la evidencia y de la investigación sobre la práctica docente; a la titulación alternativa; a la articulación con centros educativos, y a la evaluación externa. Tales categorías se ilustran con los casos de Estados Unidos y Australia, así como los de Inglaterra, Holanda, Finlandia y Suecia. Se parte del supuesto que ciertas propuestas nacionales ofrecen ideas inspiradoras para re-pensar los programas de formación docente de otras realidades educativas.

PALABRAS CLAVE: Formación docente, programas de formación, educación comparada, innovaciones en la enseñanza.

\section{Abstract}

Over the last years, an increasing number of studies and reports showing dissatisfaction with the quality of teacher training programs have appeared. Now more than ever it is necessary to ask ourselves on the importance of the improvement of the initial training of teachers, so that they can have a real performance in classrooms. For this reason, the authors propose an analysis and reflection about a series of initiatives identified in the literature. The reforms and innovations presented have been classified into five categories of analysis that refer to: the establishment of standards in training institutions, the use of evidence and research on teaching practices, the alternative qualifications, the articulation with educational centers and the external assessment. These categories are illustrated with the cases of the USA and Australia, as well as those of England, the Netherlands, Finland and Sweden. We base ourselves on the supposition that certain national proposals offer inspiring ideas to reconsider the teacher training programs in other educational realities.

KEY WORDS: Teacher education, training programs, comparative education, teaching innovation

\section{Aproximación teórica y metodológica}

Existe hoy a nivel internacional, una insatisfacción manifestada por parte de los Ministerios de Educación, de los maestros y profesores en ejercicio y de los formadores de docentes respecto a la capacidad de las universidades e institutos de formación docente, para dar respuesta a las necesidades de esta profesión (Esteve, 2000; Braslavsky, 2002; DarlingHammond, 2006; Marcelo y Vaillant, 2009). Algunos autores (Berliner, 2000) ilustran tales críticas con apreciaciones que suelen hacerse acerca de la formación inicial, tales como: "todo lo que necesita es conocimiento de la materia, el resto es una pérdida de tiempo", "cualquier persona razonablemente inteligente puede enseñar", "la mayoría de los cursos son impartidos por personas que viven en torres de marfil" o "aprender a enseñar depende tanto del contexto 
específico que no tiene sentido tratar de enseñar principios generalizables", entre otros. El autor considera que estas críticas surgen de una visión limitada de la contribución que la formación inicial tiene en el futuro desempeño de maestros y profesores. No es la formación inicial la que no sirve, sino el tipo de preparación y modalidades que se plantean para preparar buenos docentes para un adecuado desempeño profesional.

El aumento de estudios en materia de formación inicial, especialmente de organismos internacionales (Eurydice, 2002, 2004, 2006; OECD, 2004, 2009, 2011; UNESCO, 2006, 2007), se debe principalmente a las críticas en relación a la organización burocratizada de la formación; al divorcio entre la teoría y la práctica; a la excesiva fragmentación del conocimiento impartido; a la escasa vinculación con las escuelas,... (Feiman-Nemser, 2001). A esto se une el aumento de evidencias que nos indican que los programas diseñados tradicionalmente se encuentran alejados de una realidad educativa en continuo cambio. En definitiva se trata de la falta de adaptación de estos programas de formación a la gran complejidad de la sociedad del conocimiento que exige iniciativas y propuestas diversas y flexibles (Darling-Hammond y Bransford, 2005; Snow, Griffin y Burns, 2005).

Universidad y escuela deben conversar para que la formación inicial docente se desarrolle en el mejor escenario de la práctica (Valle y Manso, 2011), pero no una práctica anclada en la mera transmisión, sino una práctica profesional, comprometida con la idea de que todos somos trabajadores del conocimiento (Snoek, Uzerli, y Schratz, 2008). A nivel internacional, los nuevos desafíos de inclusión y los avances tecnológicos, han contribuido al despliegue de numerosas políticas orientadas todas ellas a mejorar la calidad de la formación de los maestros y profesores.

La formación inicial docente puede contribuir a un sistema reproductor que perpetúe inequidades múltiples o, por el contrario, puede impulsar reformas que tengan como eje, el derecho de aprender de todos los estudiantes. Y para cumplir con este último objetivo, debemos plantear la necesidad imperiosa de cambiar las estructuras organizativas y curriculares que imperan en la formación inicial de docentes (Minsun Kim, Andrews y Carr, 2004). No actuar en este sentido supone alimentar el engaño de creer que las universidades y los institutos de formación de maestros y profesores "forman" y que la escuela "deforma" (Marcelo y Vaillant, 2009).

La situación descrita hasta el momento ha sido detectada en contextos muy variados hasta tal punto que la aparición de programas globales promovidos por organismos internacionales es cada vez es mayor. Una de estas instituciones es la Organización de Estados Iberoamericanos para la Educación, la Ciencia y la Cultura (OEI) mediante el proyecto de las Metas Educativas 2021 (OEI, 2010) propuesto en el contexto iberoamericano.

El proyecto Metas Educativas 2021 (OEI, 2010) propone once finalidades subrayando la necesidad de comprometer a la sociedad con la educación. Sin embargo, se reconoce que no será posible el cumplimiento de estas metas si no se logra fortalecer, entre otros elementos, la profesión docente, en general (meta general 8), y la formación inicial en particular, (meta específica 20).

Al igual que en el ámbito iberoamericano, también en el contexto europeo comenzó a desarrollarse, como consecuencia de la Estrategia de Lisboa (European Council, 2000), el denominado programa de los Objetivos Precisos (European Commission, 2001) de cuyos 16 indicadores destinados a conseguir que todos los ciudadanos europeos participen en la nueva sociedad del conocimiento, el primero de ellos fue "Mejorar la formación (inicial y permanente) del profesorado y formadores". 
El desarrollo de proyectos como los indicados (uno ya avanzado como el programa de los Objetivos Precisos de la Unión Europea, y otro por iniciarse a través del proyecto Metas Educativas 2021) pone de manifiesto la necesidad de identificar iniciativas que apoyen la reflexión y la propuesta de mejora de los programas de formación inicial docente.

El supuesto de este artículo es que algunas iniciativas de programas de formación inicial puedan ser inspiradoras para nuevas propuestas. En este sentido, lo que se ofrece es una síntesis de información, reflexiones y sugerencias, que surgen de un amplio estudio que los autores han realizado sobre un conjunto de experiencias de formación inicial docente a nivel mundial.

El estudio comportó un proceso de identificación y categorización de experiencias significativas, entre las cuales figuran las que se presentan en este artículo. La selección de experiencias aparece marcada por el deseo de alimentar la reflexión que se está haciendo en muchos países de lberoamérica, para mejorar la preparación de maestros y profesores en sus respectivos contextos nacionales. Por otro lado, también se ha buscado reflejar la heterogeneidad de situaciones.

En una primera etapa de identificación de experiencias, se procedió a la consulta a reconocidos expertos en materia de formación docente, tanto mediante entrevistas presenciales como a través de cuestionarios escritos sobre la temática. Luego se procedió a una exhaustiva revisión de la bibliografía. Finalmente se seleccionaron las experiencias mediante una serie de criterios entre los cuales prevalecieron que, las iniciativas elegidas tuvieran al menos tres años de antigüedad y que se contara con evaluaciones e informes académicos debidamente documentados.

\section{Pistas para re-pensar la formación inicial}

El estudio que hemos realizado y especialmente la identificación de experiencias, nos ha permitido categorizar dos tipos de iniciativas que han impactado positivamente en la formación inicial docente en sus contextos de origen:

- Los estándares para la formación: la creciente autonomía brindada en muchos países a las instituciones formadoras de docentes, especialmente de las universidades, ha llevado, como contrapunto, a la necesidad en las últimas décadas del establecimiento de estándares que permitan asegurar programas de formación inicial docente de calidad (Papanastasiou, Tatto y Neophytou, 2011).

- Evidencia e investigación: La educación basada en evidencia como un componente fundamental de la formación de docentes, es otra de las tendencias que hemos observado en la revisión de la literatura (Bridges, Smeyers, y Smith, 2009). Y junto a la formación basada en evidencia, resulta también destacable el papel que la investigación debe aportar como eje central del proceso de la formación docente (Cochran-Smith y Zeichner, 2005).

Las dos categorías antes mencionadas, constituyen la base sobre la que se ha desarrollado el estudio que sintetizamos en este artículo. Adicionalmente, también consideramos tres categorías de alto interés para re-pensar los programas de formación. Éstas son:

- Mecanismos de titulación alternativa: tradicionalmente, la formación inicial de docentes se brinda en universidades o en institutos de enseñanza superior, mediante un modelo relativamente rígido, presencial y bastante alejado de los centros educativos. Sin embargo, desde hace varios años, se ha cuestionado fuertemente esa estrategia. Tal es así, que han 
surgido una serie de programas para la titulación alternativa de maestros y profesores. Se trata de mecanismos que permiten que los aspirantes a docentes puedan incorporarse a la enseñanza en un período más corto de tiempo para favorecer el reclutamiento de candidatos talentosos para la profesión (Humphrey, Wechsler y Hough, 2008).

- La articulación con los centros educativos: La tendencia en muchos países europeos es la de otorgar a las escuelas y a los centros de educación secundaria un rol preponderante en la formación inicial (Eurydice, 2012). Esta nueva forma de pensar la formación implica un cambio conceptual importante, que requiere la existencia de buenos mecanismos de selección de docentes, un sistema de evaluación por competencias muy afinado, un modelo de gestión que permita armonizar la formación con el funcionamiento de las escuelas y un marco para que la alianza entre las escuelas y las universidades pueda funcionar (Knight, Pedersen, y Peters, 2004).

- Evaluación externa: En los últimos años, los países europeos han incrementado los procedimientos de evaluación de la formación docente por parte de organismos no involucrados directamente con la misma (Eurydice, 2006). Esta evaluación externa recoge información y evidencia relacionada con las instituciones formadoras y con los programas de preparación de docentes para establecer el grado en que cumplen con determinados estándares de calidad. Normalmente, estos procesos son liderados por equipos de expertos, pares o supervisores para construir un juicio independiente en relación a la calidad de la formación ofrecida por cada programa.

Tal como mencionamos al inicio de esta sección, el estudio realizado nos ha permitido identificar una diversidad de transformaciones referidas a la formación inicial. Especialmente singular es el caso del ámbito europeo donde, a raíz de los acuerdos firmados en 1999 en Bolonia, numerosos países participantes han puesto en marcha variadas reformas de la formación inicial de docentes (Vizek Vidovic y Domovic, 2008). Los sucesivos acuerdos entre países han pautado las recientes transformaciones de la educación superior europea lo que ha provocado un impacto significativo en las instituciones formadoras y en los programas de formación docente (Eurydice, 2006, 2010; Hudson, Zgaga y Astrand, 2010).

La consulta a expertos y la revisión de la bibliografía que hemos realizado, nos ha permitido identificar, entre otras, transformaciones de interés en el Reino Unido, Holanda, Suecia y Finlandia. En esos países se están impulsando una serie de políticas concretas para mejorar la preparación de maestros y profesores, entre las que figuran: los sistemas de estandarización; los procesos de investigación como base de la formación; la titulación alternativa, la articulación entre universidades y escuelas; la presencia de evaluación externa.

También en los últimos años, y fuera del contexto europeo, otros países han generado nuevos mecanismos para la mejora de la formación inicial de docentes. En este caso, como consecuencia de nuestro estudio de bibliografía y de experiencias, destacamos los ejemplos de Australia y de los Estados Unidos, donde se ha insistido muy especialmente en el desarrollo de estándares para asegurar la calidad de los programas de formación inicial docente. Además, en el caso de los Estados Unidos, también se han implementado medidas relacionadas con la formación basada en evidencias.

Las iniciativas antes mencionadas, se sintetizan en la tabla 1 según el país de origen. Su examen en profundidad, es relevante pues podría favorecer la reflexión académica con relación a los problemas planteados en muchos contextos por la formación inicial de los docentes. 
Tabla 1. Iniciativas mundiales para mejorar la formación docente inicial

\begin{tabular}{|c|c|c|c|}
\hline & Categoría & Tipo de reforma & País \\
\hline \multirow{5}{*}{ 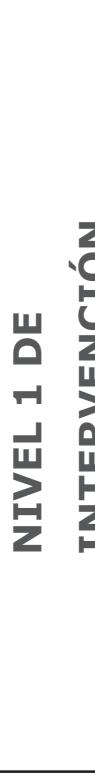 } & \multirow{3}{*}{$\begin{array}{l}\text { Estándares para la } \\
\text { acreditación }\end{array}$} & $\begin{array}{l}\text { Teacher Development } \\
\text { Agency (TDA) }\end{array}$ & Inglaterra \\
\hline & & $\begin{array}{l}\text { Evaluación en } \\
\text { base a estándares } \\
\text { en Queensland y } \\
\text { autoevaluación en el } \\
\text { Estado de Victoria }\end{array}$ & Australia \\
\hline & & $\begin{array}{l}\text { Estandarización } \\
\text { para la acreditación } \\
\text { de instituciones } \\
\text { formadoras (NCATE) } \\
\text { y aseguramiento de } \\
\text { la calidad (State of } \\
\text { California) }\end{array}$ & Estados Unidos \\
\hline & \multirow{2}{*}{$\begin{array}{l}\text { Evidencia e } \\
\text { investigación }\end{array}$} & $\begin{array}{l}\text { Docentes para una } \\
\text { nueva era }\end{array}$ & Estados Unidos \\
\hline & & $\begin{array}{l}\text { Investigación en la } \\
\text { formación y centro } \\
\text { LUMA }\end{array}$ & Finlandia \\
\hline \multirow{7}{*}{ 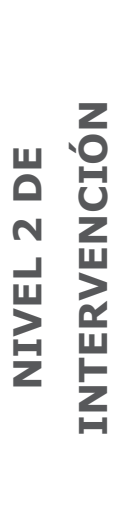 } & \multirow[t]{2}{*}{ Titulación alternativa } & $\begin{array}{l}\text { Programas de } \\
\text { certificación alternativa }\end{array}$ & Reino Unido \\
\hline & & $\begin{array}{l}\text { Rutas flexibles para la } \\
\text { formación } \\
\end{array}$ & Holanda \\
\hline & \multirow[t]{2}{*}{ Articulación con centros } & $\begin{array}{l}\text { Formación Docente } \\
\text { centrada en la escuela }\end{array}$ & Reino Unido \\
\hline & & $\begin{array}{l}\text { Alianzas con las } \\
\text { Universidades }\end{array}$ & Suecia \\
\hline & \multirow[t]{3}{*}{ Evaluación externa } & Agencia independiente & Holanda \\
\hline & & $\begin{array}{l}\text { Inspecciones de } \\
\text { educación básica }\end{array}$ & $\begin{array}{l}\text { Inglaterra, Gales e } \\
\text { Irlanda Norte }\end{array}$ \\
\hline & & Agencia educativa & Escocia y Suecia \\
\hline
\end{tabular}

Fuente: Elaboración propia

\section{Resultados}

En los párrafos que siguen presentaremos las innovaciones identificadas en la tabla 1 como potencialmente inspiradoras para re-pensar la formación inicial docente en variados contextos. Se trata de iniciativas que marcan un antes y un después en la formación inicial docente.

\section{3.a. Los estándares para la formación}

Algunos estudios demuestran la tendencia de aumento de la autonomía brindada, especialmente a las universidades, como centros formadores de maestros y profesores (Aghion et al., 2008). Este crecimiento ha hecho necesaria la regulación de esos centros por medio de los estándares.

Si examinamos el caso de Inglaterra, en el Reino Unido, el desarrollo de estándares fue favorecido por la creación en el año 1994, de una institución especializada en la formación y 
el desarrollo de los centros educativos: Teacher Development Agency (TDA). Se trata de un organismo público cuyo principal objetivo es estimular el reclutamiento de buenos candidatos para la docencia, a través de una oferta de formación de excelencia. El organismo identifica y selecciona proveedores para los cursos de formación inicial de docentes basándose en criterios y estándares especificados por la Secretaría de Estado de Educación. Luego se ocupa de monitorear si esas instituciones cumplen con los criterios y estándares establecidos. Las instituciones que imparten programas de formación docente son acreditadas según cuatro grupos de estándares:

- requisitos de ingreso para los estudiantes de los programas,

- descripción de las modalidades de formación y su evaluación,

- formas de colaboración y alianzas entre instituciones,

- mecanismos para asegurar la calidad en las instituciones formadoras.

Tabla 2. Estándares para la acreditación de las instituciones formadoras en Inglaterra

\begin{tabular}{|l|l|}
\hline Dimensión & Principales fuentes de evidencia \\
\hline Características de & $\begin{array}{l}\text { - Realización de una prueba de ingreso para estimar el nivel que el estudiante } \\
\text { locanzará al egreso } \\
\text { - Calificación mínima requerida en pruebas de lengua y matemáticas para el } \\
\text { ingreso } \\
\text { - Aprobación de exámenes físicos y psíquicos requeridos para ejercer la } \\
\text { docencia } \\
\text { - Realización de al menos una entrevista personal al momento del ingreso }\end{array}$ \\
\hline $\begin{array}{l}\text { Formación y } \\
\text { evaluación }\end{array}$ & $\begin{array}{l}\text { - Diseño e implementación de programas de formación que permitan a los } \\
\text { futuros docentes cumplir con los estándares del Qualified Teacher Status (QTS) } \\
\text { - Adecuación de los programas a las necesidades individuales de los } \\
\text { estudiantes } \\
\text { - Preparación de los estudiantes para enseñar en al menos dos niveles } \\
\text { educativos } \\
\text { - Carga horaria de 32 semanas en los programas de grado de 4 años de } \\
\text { duración y carga de 24 semanas en los programas de tres años de duración } \\
\text { - Realización de prácticas en al menos dos centros educativos para cada } \\
\text { estudiante }\end{array}$ \\
\hline $\begin{array}{l}\text { Colaboración y } \\
\text { alianzas }\end{array}$ & $\begin{array}{l}\text { - Trabajo conjunto entre las instituciones formadoras y los centros educativos, } \\
\text { tanto en la planificación de la formación, como en la selección de los estudiantes } \\
\text { y su evaluación de acuerdo a los estándares del Qualified Teacher Status (QTS) }\end{array}$ \\
\hline Calidad & $\begin{array}{l}\text { - Acceso de los estudiantes a libros, nuevas tecnologías y otros materiales } \\
\text { necesarios para la formación inicial. } \\
\text { - Revisiones periódicas de curriculum, con identificación de áreas de mejora } \\
\text { y diseño de acciones para asegurar la calidad a futuro }\end{array}$ \\
\hline
\end{tabular}

Fuente: Elaboración propia a partir de www.tda.gov.uk (último acceso: 11/05/01) 
Las reformas impulsadas en Inglaterra para la formación, desarrollo y certificación de docentes han estado centradas, por un lado, en elevar los estándares para la formación y el ejercicio de la docencia y, por otro, en reclutar y retener un número suficiente de docentes. La existencia de un organismo público que coordina la oferta de formación, ha contribuido a incrementar el número de candidatos para la enseñanza y a mejorar sensiblemente la calidad de la formación inicial.

Otro caso interesante para el análisis es Australia, donde una gran mayoría de instituciones educativas pertenece al estado y muy pocas son privadas. Australia cuenta con aproximadamente 9.000 colegios de Educación Primaria y de Bachillerato de los cuales un $70 \%$ son públicos. La administración de la educación es responsabilidad del Gobierno federal, de los estados y los territorios de Australia, quienes deciden las políticas educativas. Los dos últimos tienen la responsabilidad financiera de la educación escolar.

La formación inicial de los maestros y profesores australianos se sitúa en las universidades. Actualmente, casi 40 instituciones diferentes imparten formación de profesores en Australia. La mayoría son universidades públicas, aunque existe un pequeño grupo de instituciones privadas de educación superior que también se ocupan de la preparación inicial de docentes. Pese a sus dimensiones, no existe en el país un organismo en el ámbito nacional dedicado a evaluar y acreditar los programas de formación docente. Son los Estados y Provincias quienes tienen la responsabilidad de los procesos de acreditación cuyos avances son dispares según un relevamiento realizado hace unos años (Ingvarson et al., 2006). Esa diversidad en los sistemas de estandarización nos ha permitido identificar algunos modelos que podrían ser inspiradores para otros países.

En el estado de Queensland las regulaciones sobre registro de los docentes exigen que todos los programas de formación sean aprobados por el Queensland College of Teachers. El proceso de aprobación se basa en un conjunto de estándares estructurados en cinco grandes áreas: los conocimientos profesionales y disciplinares impartidos, el número y tipo de estudiantes, la capacidad de crear un entorno intelectualmente desafiante para los estudiantes, las relaciones profesionales y la práctica ética y los niveles de aprendizaje y de reflexión de los estudiantes (Queensland College of Teachers, 2009).

Actualmente, el proceso de aprobación de los programas de formación requiere que las instituciones formadoras provean información sobre cómo están articulando sus cursos; la evidencia empírica sobre el proceso de enseñanza-aprendizaje; las formas de impartir los cursos; la presencia de nuevas tecnologías; los vínculos entre las experiencias de aprendizaje y la evaluación; la contribución de la formación a la construcción de un perfil profesional exitoso; la retención de los estudiantes y su desempeño.

Las instituciones formadoras deben entregar un reporte anual sobre la implementación de los cursos, incluyendo transformaciones menores, y advertir a las autoridades provinciales sobre cualquier cambio sustantivo en los contenidos, las formas de impartir los cursos o la evaluación.

Otro ejemplo australiano, refiere al estado de Victoria donde desde 2004 existe un proceso de revisión y aprobación de los programas de formación docente que es administrado por el Victorian Institute of Teaching (VIT). Para acreditarse, las instituciones formadoras deben proveer información al VIT a los efectos de que éste evalúe en qué medida cumplen con los requisitos establecidos. EI VIT realiza los procesos de evaluación a través de un Comité compuesto por 25 personas, entre los cuales representantes de las ocho universidades de la Provincia que 
imparte formación docente inicial, docentes en ejercicio, padres y autoridades empleadoras de docentes. EI VIT asigna un panel evaluador para cada programa de formación que desea ser acreditado, dicho panel debe revisar el informe de auto-evaluación que cada universidad elabora (Blake y Gallagher, 2009).

Como indicábamos al inicio de esta sección, son muchos los países que registran experiencias de alto interés en materia de estándares para la formación de docentes. Además de los casos de Inglaterra y Australia, ya citados, nos interesa resaltar dos casos interesantes en Estados Unidos: The Nacional Council for Accreditation of Teacher Education (NCATE) y el sistema de aseguramiento de la calidad en la formación docente, en el estado de California.

EI NCATE, creado en 1954, opera como cuerpo independiente en la acreditación de los estándares de la formación de maestros y profesores y cuenta con el aval de las principales organizaciones profesionales de docentes e instituciones formadoras. EI NCATE acredita a instituciones formadoras de docentes con referencia a un conjunto de estándares que son revisados cada siete años en base a un amplio proceso de consulta entre educadores, tomadores de decisiones e investigadores en el campo educativo, la última de 2007. Para NCATE, los estándares miden la efectividad de las instituciones formadoras para alcanzar una formación de alta calidad. Para lograr la acreditación, una institución debe aplicar formalmente $\mathrm{y}$, a partir de este momento, se agenda una visita, luego de ello, la institución debe preparar un reporte en el que describe su situación con relación a los estándares planteados (NCATE, 2008).

Los estándares utilizados parten de una visión concreta respecto de los saberes y habilidades que los egresados de la formación docente deben tener, para trabajar en forma efectiva con sus estudiantes. En este sentido, proveen una guía que se estructura basada en seis ejes que aparecen descriptos en la tabla 3. 
Tabla 3. Estándares para la acreditación de instituciones formadoras en los Estados Unidos

\begin{tabular}{|c|c|}
\hline Estándar & Descripción \\
\hline Conocimientos y habilidades de los candidatos & $\begin{array}{l}\text { Los candidatos que egresan del programa de } \\
\text { formación tienen y demuestran conocimientos sobre } \\
\text { contenidos, entorno, prácticas pedagógicas y requisitos } \\
\text { profesionales para ayudar a todos los estudiantes a } \\
\text { aprender. Las evaluaciones indican que los candidatos } \\
\text { alcanzan los estándares estatales y los propios de la } \\
\text { institución formadora. }\end{array}$ \\
\hline Sistema de evaluación & $\begin{array}{l}\text { La institución formadora posee un sistema de } \\
\text { evaluación que recoge y analiza datos sobre las } \\
\text { calificaciones de los postulantes a los programas, } \\
\text { el desempeño de sus estudiantes y las acciones } \\
\text { institucionales que contribuyen a mejorarlo. }\end{array}$ \\
\hline Experiencia en campo y prácticas & $\begin{array}{l}\text { La institución formadora y sus centros escolares } \\
\text { asociados diseñan, implementan y evalúan experiencias } \\
\text { en campo y prácticas para que los candidatos a la } \\
\text { docencia desarrollen y demuestren los saberes, } \\
\text { habilidades y suposiciones profesionales necesarios } \\
\text { para ayudar a todos los estudiantes a aprender. }\end{array}$ \\
\hline Diversidad & $\begin{array}{l}\text { La institución formadora diseña, implementa y evalúa } \\
\text { su curriculum y provee experiencias para que todos sus } \\
\text { candidatos puedan trabajar con un alumnado diverso } \\
\text { y en distintos contextos socio-económicos. }\end{array}$ \\
\hline Calificación y desempeño de los formadores & $\begin{array}{l}\text { Los profesores están calificados y son modelos } \\
\text { de práctica profesional en el plano académico, de } \\
\text { servicio y de docencia, incluyendo la capacidad para } \\
\text { evaluar su propia efectividad y relacionarla con el } \\
\text { desempeño de sus estudiantes de formación docente. } \\
\text { También colaboran con colegas en las escuelas de } \\
\text { práctica asociadas. La institución formadora evalúa } \\
\text { sistemáticamente el desempeño de los profesores en } \\
\text { sus programas, facilitando su desarrollo profesional. }\end{array}$ \\
\hline Gobierno y recursos & $\begin{array}{l}\text { La institución formadora tiene el liderazgo, la autoridad, } \\
\text { el presupuesto, el personal, la infraestructura y los } \\
\text { recursos para que la preparación de los candidatos } \\
\text { a la docencia alcance los estándares estatales y los } \\
\text { propios de la institución formadora. }\end{array}$ \\
\hline
\end{tabular}

Fuente: Elaboración propia en base a NCATE (2008)

Los estándares antes descritos son utilizados para acreditar a las instituciones formadoras, cualquiera sean los programas que ofrezcan. Por esta razón, NCATE también ha desarrollado estándares específicos para la acreditación de los programas de formación docente, que se basa en la revisión de expertos. En la actualidad, NCATE (2008) acredita programas de formación docente en campos tan variados como "educación inicial", "educación tecnológica", "educación ambiental", "docencia de lenguas extranjeras", "ciencias básicas", "ciencias sociales" y "educación física".

El caso de California también ofrece pistas interesantes. En este estado, existen diversas modalidades de formación docente pero existe un aseguramiento y control de la calidad a través de un sistema de información que monitorea y evalúa en forma permanente a los candidatos a la docencia. La figura 1 define cuatro etapas centrales mediante las que se valora la calidad del programa de formación inicial docente. 


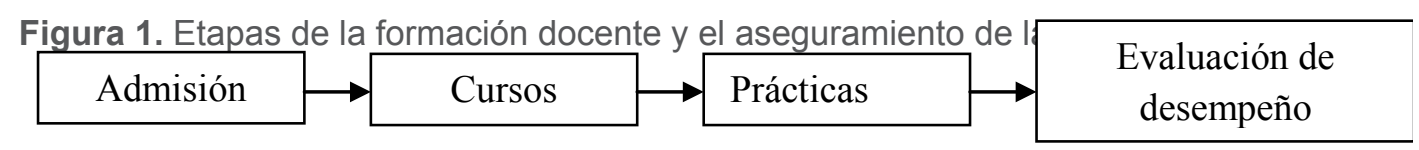

Fuente: Elaboración propia a partir de Wechsler et al. (2007)

El sistema recoge la información sobre las fortalezas y debilidades de los candidatos a la docencia en cuatro momentos clave: la admisión; el período en que se realizan los cursos; las prácticas; la evaluación de desempeño docente, al finalizar la formación. Durante la primera etapa de admisión los candidatos a la docencia son evaluados basándose en sus conocimientos con relación al área o asignatura que desean enseñar, y sus actitudes con relación a la tarea de enseñar. Para evaluar los conocimientos, se emplean las medidas estandarizadas utilizadas en el sistema universitario estadounidense (Grade Point Average - GPA) así como dos pruebas (California Basic Educational Skills Test -CBEST- y el California Subject Examinations for Teachers -CSET) que determinan el nivel de suficiencia del candidato en la asignatura específica que desean enseñar. Los test constituyen un primer filtro importante para el ingreso a la formación docente. Otra dimensión que es evaluada en la etapa de admisión, son las actitudes de los candidatos hacia la tarea de enseñar. En las entrevistas de admisión, se suele valorar la capacidad propositiva de los candidatos así como sus competencias comunicacionales y el compromiso con la docencia. Sin embargo, los resultados obtenidos en el estudio llevado a cabo por Wechsler et al. (2007) indican que esta dimensión no siempre es evaluada en forma sistemática.

Una segunda etapa se relaciona con el programa de formación que los candidatos deben completar y en la que son evaluados, a través de mecanismos que permiten establecer el grado en que poseen los conocimientos y habilidades que el estado de California, a través de las Teaching Performance Expectations (TPEs), establece que cada egresado debe tener, para poder obtener la certificación provisoria. Esas evaluaciones constituyen una herramienta analítica fundamental para sus programas, porque ofrecen la oportunidad de medir el progreso de los estudiantes hacia metas claras y precisas y, sobre todo, permiten identificar sus fortalezas y debilidades frente a distintas competencias necesarias para ejercer la docencia.

La tercera etapa del sistema de aseguramiento de la calidad de la formación docente en el Estado de California, consiste en la evaluación de las prácticas que se realizan bajo la supervisión de un docente experto y de un supervisor de la Universidad. La figura del docente experto, garantiza que quien monitorea las prácticas esté inserto en la escuela y se encuentre muy vinculado al trabajo de aula. La presencia de supervisores de la universidad asegura la presencia de criterios estandarizados para la evaluación de los programas de formación. Los supervisores universitarios, suelen no conocer la vida escolar pero conocen a la perfección los mecanismos de evaluación. Por el contrario, los docentes expertos tienen gran conocimiento de la vida de aula, pero suelen no estar muy familiarizados con los criterios de evaluación de los programas. La complementariedad entre los dos roles, asegura el buen funcionamiento del sistema.

La última etapa del sistema de aseguramiento de la calidad en el estado de California, refiere a la evaluación de desempeño al egreso del programa de formación docente. A partir del año escolar 2008-2009, todos los candidatos a la docencia deben aprobar una Teaching 
Performance Assessment (TPA), diseñada para medir el grado en que los egresados se acercan a los estándares establecidos. Existen al menos dos mecanismos (California TPA -CalTPA- y Performance Assessment for California Teachers -PACT - diseñados para evaluar un amplio espectro de habilidades y competencias para el ejercicio de la docencia. Durante estas pruebas, el estudiante debe realizar una serie de tareas referidas a la pedagogía específica de la asignatura a enseñar, al diseño de la instrucción, a la evaluación de aprendizaje y la culminación de la experiencia docente. La implementación de las pruebas es una responsabilidad de las universidades a cargo de los programas de formación docente. Para poder llevar a cabo la evaluación, las universidades se apoyan en sus profesores, supervisores, y tutores, quienes son los encargados de conducir el proceso. El Estado de California asegura la capacitación de los equipos universitarios para que estos puedan llevar adelante su tarea, a través de Commission on Teacher Credentialing encargados de supervisar y asegurar la calidad de los mecanismos de evaluación de ingreso a la profesión docente.

Para cerrar al análisis de experiencias interesantes en materia de estándares para la formación docente, vale la pena considerar el caso de Escocia. En ese país se estableció, en el año 2000, el Standard for Initial Teacher Education (General Teaching Council Scotland, 2006), que opera como un mecanismo de aseguramiento de la calidad de la formación de maestros y profesores, funcionando como el instrumento central de aprobación de los programas de formación. Los estándares fueron generados por el Comité Asesor sobre calidad de la formación docente, y establecen parámetros para la graduación de nuevos docentes. Se fijan una serie de requisitos de conocimientos y habilidades que los candidatos a la docencia deben tener al egresar de la formación, y se explicitan los requisitos que los programas deben cumplir en términos de contenidos y de metodología de trabajo.

El estándar elabora un triángulo de habilidades y competencias para los egresados compuesto por el conocimiento profesional, habilidades profesionales, y los valores profesionales y compromiso personal. A partir de allí, señala qué es lo que los docentes deben saber y ser capaces de hacer en cada dimensión.

\section{3.b. Formación basada en evidencia e investigación}

Junto al establecimiento de estándares como medida que asegure unos mínimos de calidad del periodo de formación inicial, se hace necesario igualmente desarrollar iniciativas relacionadas con características del programa. Entre ellas, el estudio realizado destaca aquellas propuestas de formación de docentes cuyo eje central es la educación basada en la evidencia y la investigación.

Un ejemplo interesante de este tipo es el proyecto Teachers for a New Era (TNE), implementado desde el año 2001 por la Carnegie Corporation of New York y que busca mejorar la efectividad del desempeño profesional docente a través de la formación inicial. Esta iniciativa, se apoya en tres supuestos fundamentales (Kirby et al., 2006):

- la formación inicial puede mejorar la calidad de la docencia,

- la mejora de la calidad en la docencia requiere asistencia externa (tanto financiera como técnica),

- el éxito de los programas puede tener un efecto multiplicador en instituciones similares.

En una primera instancia, se invita a un grupo de universidades a presentar una propuesta innovadora para su programa de formación docente. La selección de las universidades participantes en el proyecto se realiza en función de la calidad de la propuesta que presenten, y según criterios de representatividad, tamaño, antecedentes en investigación educativa y tipo 
de estudiantes formados. El programa otorga un premio de cinco millones de dólares para apoyar a cada universidad durante un período de cinco años y el financiamiento se brinda en función de una serie de principios básicos. Entre éstos figura el criterio de decisiones tomadas basándose en la evidencia, lo que implica que la transformación en los programas se realiza a partir de información resultante de una investigación continua, centrada en la medición de logros y avances de los estudiantes.

Otro de los criterios importantes refiere un equipo comprometido e integrado, y al reconocimiento de la docencia como una profesión académica que implica experiencias de práctica, lo que supone el desarrollo de alianzas duraderas entre las universidades y las escuelas para que los estudiantes de formación docente puedan conocer y experimentar la realidad de las aulas, tanto en los años de preparación como en el período de iniciación a la docencia (Kirby et al., 2006).

Las propuestas de transformación que las universidades presentan son variadas. Sin embargo, algunas evaluaciones recientes han permitido constatar que suelen incluir actividades muy similares. Para identificarlas, se han desarrollado un conjunto de indicadores, que describimos en la tabla 4.

\begin{tabular}{|l|l|}
\hline & Indicadores de actividades \\
\hline \multirow{2}{*}{$\begin{array}{l}\text { Decisiones } \\
\text { basadas en } \\
\text { evidencia }\end{array}$} & $\begin{array}{l}\text { - Se determina cuál es la información necesaria para tomar decisiones } \\
\text { - Se establece un sistema de recolección y procesamiento de la información } \\
\text { Se miden los resultados de los estudiantes }\end{array}$ \\
& $\begin{array}{l}\text { - Se realiza un seguimiento de los estudiantes a través del tiempo, identificando } \\
\text { retención, ubicación laboral, inserción } \\
\text { - El equipo docente se compromete con la construcción de la información, la } \\
\text { analiza y toma decisiones en función de ella }\end{array}$ \\
\hline $\begin{array}{l}\text { Equipo docente } \\
\text { comprometido e } \\
\text { integrado }\end{array}$ & $\begin{array}{l}\text { Los formadores de docentes proveen asesoramiento de contenidos en sus } \\
\text { asignaturas } \\
\text { - Los formadores de docentes construyen los estándares de habilidades y } \\
\text { conocimientos que los nuevos docentes deben tener } \\
\text { - Los formadores de docentes crean y revisan la estructura curricular de los } \\
\text { programas }\end{array}$ \\
\hline $\begin{array}{l}\text { Importancia de la } \\
\text { práctica }\end{array}$ & $\begin{array}{l}\text { - Se involucra activamente a los equipos docentes de las escuelas en el diseño, } \\
\text { el funcionamiento y la toma de decisiones } \\
\text { - Se crean alianzas estables entre los programas y los centros educativos de } \\
\text { práctica } \\
\text {-Se desarrollan estándares que reflejan lo que se debe esperar de las experiencias } \\
\text { de práctica } \\
\text { - Se evalúan las prácticas, y se llevan a cabo acciones para enfrentar los desafíos } \\
\text { que surgen de la evaluación }\end{array}$ \\
\hline
\end{tabular}

Tabla 4. Principios e indicadores de Teacher for a New Era

Fuente: Elaboración basada en Kirby et al., (2006)

Uno de los principales valores agregados del proyecto Teacherfor a New Era fue el énfasis en que las decisiones de transformación tomen como base la evidencia empírica. Las evaluaciones disponibles indican que todas las organizaciones participantes reconocen que la participación en el proyecto ha aumentado el interés y la preocupación por un proceso decisorio basado en evidencia empírica. En particular, el proyecto parece haber sido efectivo al generar información colectivamente construida, válida y confiable que permita evaluar los programas de formación, incluyendo los avances logrados en los aprendizajes de los candidatos a la docencia. Por 
otra parte, las evaluaciones indican que la información es utilizada efectivamente para tomar decisiones, pese a que la construcción de los datos es una tarea compleja y de largo aliento. A su vez, ha sido posible identificar un cambio cultural en las universidades que afecta ya no solo a los programas de formación docente sino a un gran número de departamentos y unidades académicas dentro de ellas (Kirby et al., 2006).

La investigación como un componente fundamental de la formación de docentes, es otra de las tendencias que hemos observado en la revisión de la literatura. Y en ese plano, Finlandia se destaca como caso ejemplar. En ese país, la formación docente se ha orientado hacia la investigación desde fines de la década del 70. Los programas de formación inicial, desde el nivel de licenciatura hasta las maestrías, tienen aproximadamente un $20 \%$ de su curricula dedicada a la investigación. También, para la obtención del título docente, los candidatos tienen que tener, como mínimo, una maestría.

Los programas de formación docente, tienen cursos y módulos referidos a métodos de investigación y sus diversas variantes (experimentación, encuestas, análisis históricos). Los cursos incluyen seminarios sobre el uso de la evidencia a lo largo del ejercicio de la profesión. Los maestros y profesores reciben una fuerte preparación en la investigación del comportamiento de niños y adolescentes. Además, la investigación está muy presente en la práctica guiada que los futuros docentes realizan en las escuelas, donde tienen la oportunidad de analizar el desempeño y la conducta de los estudiantes, el clima en el centro educativo y el relacionamiento con las familias. Deben reflexionar, extraer conclusiones y proponer líneas concretas de acción.

\begin{tabular}{|l|l|}
\hline Años & Presencia de la investigación en la formación docente \\
\hline $4-5$ & Investigación principalmente en los centros educativos municipales \\
\hline & Distintas modalidades de investigación \\
\hline & Conexión con el proyecto y la tesis de maestría \\
\hline $2-3$ & Investigación en las Universidades formadoras de docentes \\
\hline & Inicio de investigación centrada en asignaturas concretas \\
\hline & Incorporación de enfoques centrados en los estudiantes \\
\hline 1 & Formación práctica vinculada con contenidos teóricos \\
\hline
\end{tabular}

Tabla 5. La investigación y la práctica en la formación docente en Finlandia Fuente: Elaboración propia a partir de Niemi (2008)

Los profesores y supervisores de los programas de formación docente tienen la responsabilidad de guiar a sus estudiantes en trabajos de investigación concretos a lo largo de toda la carrera. El objetivo es que los futuros maestros y profesores aprendan a leer y entender investigaciones, buscar y recolectar información; analizar los datos colectados y sacar conclusiones basándose en la evidencia recogida.

La experiencia de Finlandia no sólo refleja avances en la incorporación de la investigación en la formación inicial, sino que también es pionera en la formación en servicio y el desarrollo profesional de los docentes. Un ejemplo interesante es el del Centro LUMA de la Universidad de Helsinki. Se trata de una iniciativa que conecta la investigación con el trabajo cotidiano de los docentes de ciencias, y los polos de innovación del país en empresas e industrias. El centro difunde hallazgos de investigación que puedan ser útiles para apoyar el trabajo de los docentes, pero también ofrece actualización científica en diversos campos de enseñanza. Además, 
organiza eventos de intercambio, como congresos y seminarios, y promueve el desarrollo de proyectos de investigación llevados a cabo por maestros y profesores.

\section{3.c. Mecanismos de titulación alternativa}

Cada vez son más los países en los que se han impulsado Programas de formación part-time y a distancia, apoyados en el uso de las tecnologías de la información y la comunicación (TIC).

Entre otros, podemos citar el caso del Reino Unido donde se han desarrollado modelos de formación del profesorado a tiempo parcial, que complementan la oferta clásica de formación existente (Eurydice, 2002; European Parliament, 2008). El Graduate Teacher Programme (GTP) y el Registered Teacher Programme (RTP) son iniciativas a tiempo parcial, para formar graduados de diversas áreas, que trabajan en centros educativos. Los mencionados programas preparan para el ejercicio docente a personas con títulos terciarios que trabajan en la enseñanza. Tienen una duración aproximada de un año, aunque los candidatos que tienen más experiencia docente pueden completarlo en menos tiempo.

Además del ejemplo del Reino Unido, podemos citar el caso de Holanda, donde los programas universitarios comenzaron y deben en la actualidad obligatoriamente incorporar rutas flexibles para la formación inicial. Es así que se pueden combinar estudios terciarios generales con la formación específica para la docencia. Los futuros maestros o profesores, pueden cursar los dos últimos años de una licenciatura universitaria con un año adicional en formación docente específica. A partir del año 2000, han proliferado los programas de certificación alternativa que intentan identificar y reclutar nuevos candidatos para la docencia. Estos programas incluyen a docentes graduados que han abandonado el ejercicio de la enseñanza, pero desean volver a ejercerla, y también a profesionales de otras disciplinas a quienes interesa integrarse a la docencia. El objetivo de incorporar en menos tiempo las competencias y conocimientos para poder enseñar, parece haberse cumplido, ya que los estudios disponibles dan cuenta de los muy buenos resultados obtenidos por los docentes certificados por rutas alternativas (Brouwer, 2007).

\section{3.d. La articulación con los centros educativos}

Al igual que en apartado anterior, son también numerosos los países que han desarrollado estímulos para que, en alianza con las universidades, los centros educativos se conviertan en instituciones proveedoras de formación docente (Walkington, 2007).

En el Reino Unido, las instituciones de educación superior que deseen ofrecer programas de formación inicial de docentes deben asociarse con escuelas para organizar un programa de formación. Por otra parte, los centros educativos tienen la posibilidad de convertirse individualmente en instituciones formadoras de profesorado a través de la School Centred Initial Teacher Training. Las escuelas y sus docentes, son seleccionadas como colaboradoras con criterios de calidad y pasan a formar parte de un consorcio al que los graduados universitarios interesados pueden, a su vez, optar a ser seleccionados para participar en dicho programa. El compromiso de la escuela con los participantes suele ser de un año de duración. Por medio de estos cursos los participantes obtienen el QTS (Qualified Teacher Status) y en la mayoría de los casos también un certificado de postgrado en educación. El convenio por el cual las escuelas son aceptadas como colaboradas del proceso de formación inicial se coordina por medio de la agencia que regula la formación docente en el país, la TDA.

En Suecia también se ha generando un sistema que premia a las instituciones formadoras que logran buenas alianzas con las escuelas en sus programas de formación inicial. Se trata 
de un sistema de alianzas entre instituciones de formación inicial y centros de enseñanza básica para promover un mayor involucramiento de estos últimos en la formación de profesores (Ministry of Education and Science of Sweden, 2003). La característica más significativa de estas propuestas es el énfasis en la inmersión de los estudiantes en formación en equipos de trabajo coordinados y constituidos por profesores de la universidad y de los centros. Esto favorece la construcción de consensos y marcos compartidos entre el ámbito académico de la universidad y el desarrollo práctico de los centros educativos que eleva significativamente la calidad, especialmente, de la formación inicial de los futuros docentes.

\section{3.e. Evaluación externa}

Para muchos países europeos la evaluación externa es un requisito formal de los sistemas de formación docente y es implementada por una agencia evaluadora, comité o cuerpo independiente que actúa en representación de las autoridades públicas.

Este es el caso de Holanda con la excepción de que realiza un proceso mixto ya que la evaluación externa la realizan tanto un comité de evaluación como un organismo independiente que de forma coordinada realizan los procedimientos de evaluación externa. También está el caso del Reino Unido (Inglaterra, Gales e Irlanda del Norte), donde la formación docente inicial tampoco responde a los procedimientos comúnmente utilizados en la Unión Europea sino que en este caso se opta por una evaluación externa realizada por las inspecciones de educación básica. Por último, especialmente significativo son los casos de Escocia y Suecia. Son los único países en los que se ha optado por que sea una agencia educativa la que lleve a cabo la evaluación externa (Eurydice, 2006).

Teniendo en cuenta las iniciativas presentadas consideramos necesario ofrecer dos principales reflexiones de sus evaluaciones externas en relación con los programas de formación inicial del docente.

Comenzaríamos indicando que la evaluación externa tiene diverso alcance y se refiere, por lo general, al contenido curricular de los programas de formación, a los métodos de enseñanza o a las prácticas de evaluación de maestros y profesores. Puede también considerar el tiempo ocupado por los contenidos disciplinares y por los específicamente pedagógicos; las modalidades de gestión y las características de las instituciones formadoras; las alianzas potenciales entre éstas y las escuelas; los recursos humanos empleados. Otros aspectos importantes que pueden ser evaluados son el desempeño de los estudiantes, sus actitudes y motivaciones, sus opiniones sobre la preparación que están recibiendo, y la infraestructura general de las instituciones formadoras (incluyendo bibliotecas, acceso a nuevas tecnologías...).

Y por otro lado, la puesta en marcha de procesos de evaluación externa se debe acompañar de una serie de debates referidos entre otros aspectos, a los propios procedimientos de evaluación ya que la apreciación externa de los procesos de formación docente implica contar con una base de información que no siempre está disponible. Además, la evaluación externa requiere un marco referencial con las capacidades y competencias que debe tener un buen docente, lo que a menudo no existe de manera explícita. En esta misma línea, otro de los temas polémicos que conviene tener en cuenta, es la utilización de los hallazgos de las evaluaciones externas en los procesos de decisión que pautan la acreditación o re-acreditación de instituciones.

\section{Discusión}

Los docentes importan, e influyen mucho en el aprendizaje de los estudiantes y en la mejora 
de la calidad de la educación. Y puesto que los maestros y profesores cuentan, necesitamos que nuestros sistemas educativos sean capaces de atraer a los mejores candidatos para convertirlos en docentes. Necesitamos buenas políticas para que la formación inicial de esos maestros y profesores les asegure las competencias que van a necesitar a lo largo de su extensa trayectoria profesional. Y la sociedad requiere de buenos docentes cuya práctica profesional asegure los estándares profesionales de calidad y garantice el derecho de los estudiantes a aprender.

En este artículo, hemos intentando evidenciar, que existen hoy experiencias a nivel internacional que cumplen con los requisitos planteados por Fullan hace más de una década atrás (1998) cuando indicaba que para reconstruir la formación docente sería necesario generar una base de conocimiento mayor para la docencia y atraer estudiantes capaces, diversos y comprometidos con la profesión. Para alcanzar esos objetivos, el autor planteaba rediseñar los programas de formación para fortalecer los vínculos entre las artes y las ciencias y entre la teoría y la práctica; reformar las condiciones de trabajo en las escuelas; desarrollar y monitorear estándares externos para los programas y desarrollar investigación rigurosa y dinámica centrada en la docencia.

La categorización de experiencias realizada en nuestro estudio, han sido ilustradas por una serie de casos que evidencian que es posible transformar la formación inicial de docentes. Los casos expuestos de Australia, Estados Unidos, Finlandia, Holanda, Reino Unido y Suecia, adoptan diversos caminos que pasan por la determinación de estándares que permitan retroalimentar las propuestas de formación que deben, a su vez, basarse en explícitas evidencias sobre el aprendizaje; la enseñanza y la investigación. Además se establecen también como elementos importantes la articulación con las escuelas, las titulaciones alternativas y la evaluación externa.

Un análisis más preciso de estas iniciativas nos permite identificar características más específicas sobre las que centrar la reflexión. En primer lugar, las experiencias analizadas parecen remitir a tópicos que, de una u otra forma, responden a las necesidades educativas propias de las transformaciones que experimentan las sociedades contemporáneas. Hoy el mundo ha cambiado y la preparación de docentes debe contemplar tendencias como la sobreabundancia de información, o la globalización de las comunicaciones.

Además, las experiencias analizadas ponen de manifiesto la centralidad de las escuelas y de los estudiantes como condición crítica de la efectividad de las iniciativas. Es el centro educativo el telón de fondo de la formación y el eje articulador en torno al cual se construyen las diversas actividades de preparación para un adecuado desempeño de los futuros docentes.

Otro aspecto importante a destacar, es que las experiencias estudiadas promueven el desarrollo autónomo de los docentes, alejándose de lo que Gautier considera "una visión paternalista de la formación y capacitación de los maestros" (2005: 16). Y esto tiene que ver con otra de las tendencias que hemos identificado y que se vincula con el trabajo colaborativo y con el desarrollo de capacidades para trabajar en equipo. La visión compartida y la articulación entre los actores implicados en las experiencias formativas; aparece como un elemento que favorece la puesta en marcha de procesos que promuevan un mejor desempeño de los futuros docentes.

Por último, nos parece necesario agregar la importancia de considerar a la innovación como un valor constituyente de las experiencias de formación analizadas. Se trata de iniciativas que 
incluyen elementos innovadores tanto en el diseño como en los procesos de implementación del periodo formativo.

En este artículo se han reseñado las características técnicas de una serie de experiencias vinculadas con la formación inicial de docentes, partiendo del supuesto de que éstas pueden ser inspiradoras para otros contextos. En estudios ulteriores habría que profundizar en análisis que den cuenta de si -en otros contextos- sería posible una implementación total o parcial de tal tipo de innovaciones.

No se trata sólo de la factibilidad política, económica, social, histórica o cultural de las experiencias estudiadas en este artículo sino también de la consideración de las cuestiones inherentes a la implementación específica de cambios y de su eventual transferibilidad a otros contextos. Y es que, por encima de estas nuevas iniciativas, para el éxito de la formación inicial son necesarias políticas sistémicas y con sostenimiento en el tiempo, que consideren la multiplicidad de factores que intervienen en la compleja tarea de aprender y de enseñar. No alcanzan las buenas intenciones, ni las declaraciones, ni las meras palabras. Los sistemas educativos deben transformarse al ritmo de los cambios que se están operando en los sociales y económicos: ser cada vez más incluyentes y dar respuesta al derecho de aprender de todos los niños y adolescentes.

\section{Bibliografía}

Aghion, P., Dewatripont, M., Hoxby, C., Mas-Colell, A., y Sapir, A. 2008. Higher aspirations: An agenda for reforming european universities. Brussels: Bruegel.

Berliner, D. C. 2000. A personal response to those who bash teacher education. Journal of Teacher Education, 51(5), 358-371.

Blake, D., y Gallagher, D. 2009. Examining the Development of the Victorian Certificate of Applied Learning and Its Implications for Schools and Teacher Education in Australia. Journal of Applied Learning in Higher Education, 1, 49-72.

Braslavsky, C. 2002. Current teacher education from the perspective of the demands of curricular change. In 54th Annual Meeting of the American Association of Colleges for Teacher Education. Recuperado de http://www.ibe.unesco.org/fileadmin/user_upload/Organization/ Director/AACTE2002

Bridges, D., Smeyers, P., y Smith, R. 2009. Evidence-based education policy: What evidence? What basis? Whose policy? London: Wiley-Blackwell.

Brouwer, N. 2007. Alternative teacher education in the Netherlands 2000-2005. A standards based synthesis. European Journal of Teacher Education, 30(1), 21-40.

Cochran-Smith, M. y Zeichner, K. 2005. Studying teacher education: The report of the AERA panel on research and teacher education. Mahwah, $\mathrm{NJ}$ : Lawrence Erlbaum Associates Inc.

Darling-Hammond, L. y Bransford, J. 2005. Preparing teachers for a changing world: what teachers should learn and be able to do. San Francisco: National Academy of Education.

Darling-Hammond, L. 2006. Constructing 21st-Century Teacher Education. Journal of Teacher Education, 57 (3), 300-314.

Esteve, J.M. 2000. The Transformation of Teachers' Role at the end of Twentieth Century. New Challenges for the Future. Educational Review, 52 (2), 197-208. 
European Commission. 2001. Report from the Commission - The concrete future objectives of education systems. Brussels: Comission of the European Communities.

European Council. 2000. Presidency conclusion: Lisbon European Council - 23 and 24 march. Brussels: Council of the European Council.

European Parliament. 2008. Culture and education. Content and quality of teacher education across the European Union. United Kingdom: Institute of Education, University of London.

Eurydice. 2002. The teaching profession in Europe: Profile, trends and concerns. General lower secondary education. Initial training and transition to working life. Volume 1. Brussels: Directorate-General for Education and Culture.

Eurydice. 2004. The teaching profession in Europe: Profile, trends and concerns. General lower secondary education. Keeping teaching attractive for the 21 st century. Volume 4. Brussels: Directorate-General for Education and Culture.

Eurydice. 2006. Quality assurance in Teacher Education in Europe. Brussels: DirectorateGeneral for Education and Culture.

Eurydice. 2012. Key Data on Education in Europe 2012. Brussels: Education, Audiovisual and Culture Executive Agency.

Eurydice. 2010. Focus on Higher Education in Europe 2010: The impact of the Bologna Process. Brussels: Education, Audiovisual and Culture Executive Agency.

Feiman-Nemser, S. 2001. From Preparation to Practice: Designing a Continuum to Strengthen and Sustain Teaching. Teachers College Record, 103 (6), 1013-1055.

Fullan, M. 1998. The meaning of educational change: A quarter of a century of learning. En A. Hargreaves, A. Lieberman, M. Fullan y D. Hopkins (eds.), International Handbook of Educational Change (pp. 214-228). Dordrecht: Kluwer.

General Teaching Council Scotland. 2006. The Standard for Initial Teacher Education. Edinburgh: GTCS.

Gautier, E. 2005. Nuevas tecnologías y formación docente: análisis de experiencias relevantes en América Latina. En M. Robalino y A. Körner Formación docente y las tecnologías de información y comunicación. Estudios de casos en Bolivia, Chile, Colombia, Ecuador, México, Panamá, Paraguay y Perú. Santiago de Chile: OREALC- UNESCO.

Hudson, B., Zgaga, P., y Astrand, B. 2010. Advancing quality cultures for teacher education in Europe: Tensions and Opportunities. Ljubljana: Umeå School of Education, Umeå University.

Humphrey, D.C., Wechsler, M.E., y Hough, H.J. 2008. Purchase Characteristics of Effective Alternative Teacher Certification Programs. Teachers College Record. 110 (1), 1-63.

Ingvarson, L., et al. 2006. Teacher education accreditation: A review of national and international trends and practices. Recuperado de http://research.acer.edu.au/cgi/ viewcontent.cgi?article=1000ycontext=teacher_education

Kirby, S., McCombs, J., Barney, H., y Naftel, S. 2006. Reforming teacher education: something old, something new. Santa Monica-CA: Rand Corporation.

Knight, S.L., Pedersen, S., y Peters, W. 2004. Connecting the University with a Professional 
Development School: Pre-service Teachers' Attitudes Toward the Use of Compressed Video. Journal of Technology and Teacher Education, 12(1), 139-155.

Marcelo, C. y Vaillant, D. 2009. Desarrollo Profesional Docente. ¿Cómo se aprende a enseñar? Madrid: Narcea.

Minsun Kim, M., Andrews, R.L. y Carr, D.L. 2004. Traditional versus Integrated Preservice Teacher Education Curriculum: A Case Study. Journal of Teacher Education, 55(4), 341-356.

Ministry of Education and Science of Sweden. 2003. Attracting, Developing and Retaining Effective Teachers. Background report prepared for the OECD Thematic Review of Attracting, Developing and Retaining Effective Teachers. Stockholm: EQ-print.

NCATE. 2008. Professional standards. Accreditation of Teacher Preparation Institutions. Washington D.C.: NCATE.

Niemi, H (2008). Advancing Research into and during Teacher Education. En Hudson, B. y Zgaga, P (Eds.), Teacher education policy in Europe: a Voice of Higher Education Institutions. A voice of Higher Education Institutions (pp. 183-208). Ljubljana: Universities of Umea and Ljubljana.

OECD. 2004. Attracting, Developing and Retaining Effective Teachers - Final Report: Teachers Matter. Paris: OECD Publishing.

OECD. 2009. Creating Effective Teaching and Learning Environments: First Results from TALIS. Paris: OCDE Publishing.

OECD. 2011. Building a high Quality Teaching Profession. Lessons from around the World. Paris: OECD Publishing.

OEI. 2010. Metas Educativas 2021: la educación que queremos para la generación de Ios bicentenarios. Madrid: OEI.

Papanastasiou, E.C., Tatto, M.T., y Neophytou, L. 2011. Programme theory, programme documents and state standards in evaluating teacher education. Assessment y Evaluation in Higher Education, doi: 10.1080/ 02602938. 2010. 534760

Queensland College of Teachers. 2009. Professional Standards for Queensland teachers (graduate level): A guide for use with pre-service teachers. Queensland: Queensland College of Teachers.

Snoek, M., Uzerli, U., y Schratz, M. 2008. Developing Teacher Education Policies through Peer Learning. En B. Hudson y P. Zgaga (eds.), Teacher Education Policy in Europe. A voice of Higher Education Institutions (pp. 135-156). Ljubljana: Universities of Umea and Ljubljana.

Snow, C.E., Griffin, P., y Burns, M. S. 2005. Knowledge to support the teaching of reading: preparing teachers for a Changing World. San Francisco: National Academy of Education.

UNESCO. 2006. Modelos innovadores en la formación inicial docente. Santiago de Chile: UNESCO.

UNESCO. 2007. Good Practices in Education for Sustainable Development: Teacher Education Institutions. Paris: UNESCO.

Valle, J.M., y Manso, J. 2011. La nueva formación inicial del profesorado de Educación Secundaria: modelo para la selección de buenos centros de prácticas. Revista de Educación, 354 (1), 263-290.

Vizek Vidovic, V., y Domovic, V. 2008. Researching Teacher Education and Teacher Practice: the Croatian Perspective. En B. Hudson y P. Zgaga (eds.), Teacher Education Policy in 
Europe. A voice of Higher Education Institutions (pp. 303-312). Ljubljana: Universities of Umea and Ljubljana.

Walkington, J. 2007. Improving partnerships between schools and universities: professional learning with benefits beyond pre-service teacher education. Teacher Development, 11 (3), 277-294.

Wechsler, M., Tiffany-Morales, J., Campbell, A., Humphrey, D., Kim, D., Shields, P., y Wang, H. 2007. The Status of the Teaching Profession 2007. Santa Cruz, CA: The Center for the Future of Teaching and Learning.

* Fecha de recibido: 30/01/2012

Fecha de aceptado:12/04/2012

* Ph.D. en Educación, Université du Québec, Canadá. Master en Planificación y Gestión, Université de Genéve, Suiza. Licenciada en Ciencias de la Educación. Especialista en planificación y gestión de sistemas educativos y en formación docente. Miembro, Board del International Council on Education for Teaching. Presidenta, Comité Científico del Observatorio Internacional de la Profesión Docente. Secretaria Académica, Instituto de Educación, Universidad ORT Uruguay. Directora del Doctorado y Master en Educación. Investigador Nivel II - Sistema Nacional de Investigadores (SNI).

${ }^{* *}$ Realiza su tesis doctoral sobre formación inicial del profesorado de Educación Secundaria, Departamento de Didáctica y Teoría de la Educación, Universidad Autónoma de Madrid (UAM). Máster en Mejora y Calidad de la Educación. Licenciado en Psicopedagogía (por lo que obtuvo el Premio Nacional a la excelencia académica). Maestro en Educación Especial. Miembro del equipo directivo de la Asociación de Jóvenes Investigadores en Teoría e Historia de la Educación y Coordinador de áreas del Grupo de Investigación sobre Políticas Educativas Supranacionales, Universidad Autónoma de Madrid. Investigador en el Profesorado de Educación Secundaria y en Política Educativas. 